\title{
Avaliação do impacto do Programa Saúde da Família no perfil epidemiológico da população rural de Airões, município de Paula Cândido (M G), 1992-2003
}

\author{
Evaluation of the impact of the Family Health Program \\ in the epidemiology profile of the rural population of Airões, \\ city of Paula Cândido (M G), 1992-2003
}

\author{
Karine de Oliveira Gomes ${ }^{1}$ \\ Rosângela M inardi M itre Cotta ${ }^{1}$ \\ MarilenePinheiro Euclydes ${ }^{1}$ \\ Carina Loureiro Targueta ${ }^{1}$ \\ Silvia Eloíza Priore ${ }^{1}$ \\ Sylvia do Carmo Castro Franceschini ${ }^{1}$
}

${ }^{1}$ Departamento deN utrição eSaúde, Universidade Federal deViçosa. Av. Peter Henry Rolfs $s / n$, Campus Universitário. 36570-000 Viçosa MG.

kogomes@yahoo.com.br
Abstract The Family Health Program (PSF) as a redirection strategy of the healthcare model of the country has promoted the substitution of assistential and cure model for the disease prevention and health promotion of individuals as well as the families and communities, placing the primary health care as the entrance to the healthcare system. This work aims to evaluate the PSF impacting in the rural community of Airões, in the city of Paula Cândido, M inas Gerais State, analyzing its contribution to the evolution of health indicators, since the comparison of sanitary data before and after implementation of the program, in the period ranging from 1992 to 2003. The information were obtained through a household survey applied to 127 families randomly selected within the community, repre senting $55.2 \%$ and $41.6 \%$ of the families in $1992 \mathrm{e}$ 2003 respectively. After thePSF implementation there were a increase of $40,4 \%$ in diseases diagnosis and a reduction in the number of internment from $42.7 \%$ to $27.2 \%$. Prenatal assistance coveragewas enlarged from $30.6 \%$ to $90 \%$ and the birth in homes reduced from $48.9 \%$ to $1 \%$. Therefore, the evolution of the sanitary data in the community shows the characterization of the PSF as an effective strategy of implementation of the U nified $\mathrm{H}$ ealth System.

Key words Primary health care, Unified Health System, Family H ealth Program, Program evaluation, Indicators
Resumo O Programa Saúde da Família (PSF), como estratégia de reorientação do modelo de atenção à saúde no país, tem promovido a substituição do modelo assistencialista e centrado na cura pela prevenção de doenças e promoção da saúde de indivíduos, famílias e comunidades, colocando a atenção básica como a porta de entrada no sistema de saúde. Este artigo tem como objetivo avaliar o impacto do PSF na comunidade rural de Airões, município de Paula Cândido, M inas Gerais, analisando sua contribui ção para a evolução dos indicadoresde saúde, a partir da comparação dos dados sanitários antes e depois da implantação do programa, no período de 1992 e2003. Osdados foram coletados por meio de inquérito domiciliar, aplicados a 127 famílias selecionadas aleatoriamente na comunidade, querepresentavam $55,2 \%$ e $41,6 \%$ do total de famílias em 1992 e 2003, respectivamente. A pós a implantação do PSF, houve um aumento de 40,4\% no diagnóstico de doenças e o número de internações reduziu de $42,7 \%$ para $27,2 \%$. A cobertura da assistência pré-natal aumentou de 30,6\% para 90\% e os partos domiciliares reduziram de $48,9 \%$ para $1 \%$. Desta forma, a evolução dos dados sanitáriosda comunidade reforça a caracterização do PSF como uma estratégia eficientede implementação do Sistema Único de Saúde.

Palavras-chave Atenção básica, SUS, PSF, Avaliação de programas e projetos de saúde, Indicadores 
Introdução

O Programa Saúde da Família (PSF) surge no ce nário brasileiro como estratégia de reorientação do modelo assistencial, substituindo o modelo tradicional de assistência à saúde, historicamente curativo e hospitalocêntrico, por um modelo sintonizado com os princípios do SistemaÚ nico deSaúde (SUS), tais como a universalidade, equidade, hie rarquização e integralidade da atenção ${ }^{1}$.

Baseado numa nova dinâmica de organização dos serviços e ações de saúde, o programa tem como principais características a adscrição da clientela, através da definição de território de abrangência da equipe; enfoque sobre a família, a partir de seu ambiente físico e social como unidade de ação; estruturação de equipe multiprofissional; atividade preventiva em saúde; a detecção de ne cessidades da população e a atuação intersetorial visando à promoção da saúde ${ }^{2,3}$.

Além disso, ao longo dos doze anos de implementação, vem contribuindo com a diminuição das iniquidades, sobretudo a partir da ampliação do acesso aos serviços básicos de saúde e da vinculação das equipes junto aos indivíduos, famílias e comunidades na complexa tarefa de cuidar da vida, saúde, doença, sofrimento e morte4.

Evidências recentes têm mostrado que o PSF, além da expansão das ações e da cobertura do serviço, vem produzindo impacto sobre os níveis de saúde, reduzindo de forma significativa as taxas de mortalidade infantil, tanto a pós-neonatal como a neonatal ${ }^{5}$.

Em Sobral, o PSF foi implantado em 1997, privilegiando a atenção materno-infantil, a reorganização da assistência ao pré-natal, ao parto e nascimento, assi $m$ como a vigilância à saúdeno primeiro ano de vida. 0 trabal ho integrado e humanizado dos profissionais do programa permitiu a realização de ações de promoção à saúde e de prevenção das doenças mais prevalentes na infância, possibilitando redução na taxa de mortalidade infantil enas taxas deinternação por desnutrição, assim como o aumento da cobertura pré-natal, do número de crianças em aleitamento materno exclusivo e da cobertura de imunização ${ }^{6}$.

Nos municípios do Estado do Rio de Janeiro, considerando a hipertensão arterial, efeitos nítidos da prevenção realizada pela Estratégia Saúde da Família foram verificados pelas coberturas superiores a 70\% de medida de pressão arterial, independentemente do contexto social do município, resultando na homogeneidade do diagnóstico e dos cuidados assistenciais com a doença?.
Pesquisas realizadas pelo M inistério da Saúde verificaram que as ações educativas foram incorporadas pelas equipes, mas em patamares queainda necessitam ser incrementados; além disso, os principais problemas encontrados para a implementação do PSF foram a dificuldade de substituição do modelo e da rede tradicional de atenção à saúde, a inserção e desenvolvimento de recursos humanos e o monitoramento efetivo do processo e seus resultados, além da garantia da integralidade aos demais níveis de complexidade da rede de serviços de saúde ${ }^{2,3}$.

Neste sentido, estudos observacionais criteriosos podem ser vantajosos na avaliação de intervenções em saúde pública, tanto pelo estabelecimento de grupos de comparação e realização de medidas padronizadas quanto pela utilização de critérios bem definidos para julgar os resultados. Desse modo, estudos desta natureza são recomendáveis na avaliação de políticas e programas de saúde, pois permitem maior capacidade de detectar o sucesso ou a falha da intervenção, aumentado a validade dos resultados ${ }^{8}$.

Considerando a importância da avaliação na implementação de todo e qualquer modelo assistencial ou mesmo estratégia de atenção, como éo caso do $\mathrm{PSF}^{9}$, e que os inquéritos populacionais de saúde vêm sendo utilizados de forma crescente como meio de obtenção de informações para este $\mathrm{fim}^{7,10}$, o objetivo deste estudo foi avaliar o impacto do PSF no perfil epidemiológico da população rural de Airões, Paula Cândido, M inas Gerais.

A partir da comparação dos dados sanitários antes e depois da implantação do PSF, referentes aos anos de 1992 e 2003, respectivamente, pretende-se problematizar os principais desafios e apontar alternativas para a efetiva consolidação da estratégia em distintos contextos locais e regionais.

\section{Metodologia}

\section{Características da área de estudo}

Paula Cândido possui apenas o distrito sede e a população rural está distribuída de forma dispersa pelo território municipal. A comunidade de Airões encontra-se a onze quilômetros do município e, em 1992, contava com uma população aproximada de 1.500 habitantes, distribuídos em 230 domicílios. Já em 2003, possuía em torno de 1.800 habitantes e 305 domićlios ${ }^{11,12}$.

O município está localizado na região da Zona da M ata M ineira e apresenta estrutura fundiária, 
predominando pequenos produtores e trabalhadores rurais assalariados que desenvolvem atividades agropecuárias temporárias e sazonais, como o cultivo delavouras de milho, feijão, café, goiaba, entre outras, gerando baixo nível derenda aos seus habitantes ${ }^{11,13}$.

Segundo o censo demográfico de 2000, apenas $41,1 \%$ das famílias do município possuíam rendimento deatéum salário mínimo e 37,7\% não apresentavam rendimento mensal ${ }^{13}$.

Desenho do estudo

Este trabalho é parte de uma pesquisa desenvolvida pelo Departamento de Nutrição e Saúde (DNS) da Universidade Federal de Viçosa (UFV), que investigou as condições de vida, saúde e nutrição de al gumas famílias da comunidade de Airões, nos anos de 1992 e 2003, visando avaliar o serviço local de saúde para subsidiar a criação e implementação de políticas direcionadas aos principais problemas da população, assim como orientar as ações para 0 alcance de uma gestão e alocação de recursos mais eficiente.

Trata-se de um estudo descritivo de delineamento transversal, no qual foram entrevistadas 127 famílias da comunidade rural de Airões, nos anos de 1992 e 2003. 0 inquérito domiciliar foi 0 instrumento escolhido para a coleta dos dados sobre as condiç̧̃̃es sociais, econômicas, de saúdee nutrição da população, sendo que o mesmo inquérito foi aplicado nos dois períodos de investigação, porém para amostras distintas.

Atualmente, os inquéritos domiciliares são reconhecidos como importantes fontes de informação em saúde, constituindo uma fonte primária de dados primordiais para o estudo das desigualdades no estado de saúde e para a avaliação do acesso, da utilização e da capacidade de resolução dos serviços de saúde. Além disso, na perspectiva de se conhecer o perfil epidemiológico da população, independentemente da procura de assistência ou de informações obrigatórias, os inquéritos são ferramentas necessárias e complementares às informações rotineiramente coletadas pelos diversos sistemas de informação 7,10 .

A amostra foi selecionada segundo a base de amostragem dos cadastros censitários do município, de forma probabilística e aleatória, tanto em 1992 quanto em 2003, seguindo os critérios recomendados por Richardson ${ }^{14}$, uma vez que a seleção guiada pelas leis da probabilidade e por fórmulas apropriadas para o cálculo do tamanho da amostra estabelece uma chance adequada de ser representativa da população.
No estudo de 1992, o cálculo do tamanho da amostra foi realizado através da fórmula amostras al eatórias simples para universos finitos, considerando um nível de confiança de $95 \%$ eum erro de estimação de $5 \%{ }^{15}$, cujo resultado definiu uma amostra de 146 famílias. Entretanto, algumas famílias se negaram a participar do estudo e alguns questionários tiveram queser excluídos da análise, tendo sido avaliadas 127 famílias, que correspondiam a $55,2 \%$ do total de famílias da comunidade neste período ${ }^{11}$.

Em 2003, tendo como referencia os recursos disponíveis para a realização da pesquisa, a equipe optou por trabal har com o mesmo número de famílias de 1992, de forma que a amostra representou $41,6 \%$ da população ${ }^{12}$, sendo que todas as famílias sorteadas e entrevistadas estavam cadastradas no PSF.

Como a comunidadenão apresentou um crescimento populacional muito acentuado entre 0 período de investigação, muitas famílias foram entrevistadas nos dois momentos do estudo. Para responder ao questionário, foram estabelecidos alguns critérios, tais como residir no domicílio e estar presente no momento da entrevista alguma pessoa com idade acima de dezoito anos.

Para a avaliação do PSF, foram selecionadas as informações relatadas pelo entrevistado (a) sobre morbidades e óbitos ocorridos na família, cobertura da assistência pré natal e características de nascimento dos filhos, de forma que o impacto do programa foi avaliado pela evolução dos indicadores de saúde da população, a partir da comparação dos dados sanitários de 1992, quando ainda não havia o PSF, com os dados sanitários de 2003, após a implantação do programa.

As informações foram processadas e avaliadas com o auxílio do software Epi Info (versão 6.04) e as análises foram realizadas através da frequência das variáveis.

A pesquisa teve aprovação do Comitê de Ética em Pesquisas com Seres Humanos da Universidade Federal de Viçosa, em conformidade com os princípioséticos recomendados pela Resolução 196/ 96 do Conselho Nacional de Saúde.

\section{Resultados}

Evolução do Sistema M unicipal de Saúde

Em 1992, o município de Paula Cândido possuía um hospital com 25 leitos e um centro de saúde que contavam [é isso mesmo? o hospital E o centro contavam com cinco médicos?] com o ser- 
viço de cinco médicos. As ações de saúde eram orientadas por um modelo assistencial curativo, individual e baseado na concepção tradicional de saúde como ausência de doença ${ }^{11}$.

Por consequência, a estrutura hospitalar era utilizada para a atenção primária e tratamento dos problemas de saúde, sem levar em consideração seus fatores determinantes e o princípio da regionalização, o que associado à ausência de planejamento dificultava o acesso e prejudicava a resolutividade das ações desenvolvidas ${ }^{12}$.

Neste período, a comunidade de Airões já possuía um posto de saúde que oferecia à população medicamentos, aplicação de injeções, real ização de curativos e vacina para crianças, quando havia os recursos necessários para a realização de tais atividades. 0 posto ficava sob a responsabilidade de um agente de saúde e 0 atendimento médico ambulatorial ocorria de forma esporádica ${ }^{11}$.

Em 1998, o município iniciou o processo de inversão do sistema de atenção e estabeleceu a implantação do PSF como estratégia norteadora para a atenção básica, al cançando em 2003 a Gestão Plena da Atenção Básica. N este mesmo ano, o município contava com três equipes de Saúde da Família, sendo duas na zona rural e uma na zona urbana, distribuídas em dezoito microrregiões ecobrindo $92 \%$ da população do município ( 2.495 famílias e 9.111 pessoas), com cadastro através de prontuários individuais e familiares ${ }^{12}$.

Cada microrregião era acompanhada por uma equipe básica composta por um médico, um dentista, um enfermeiro, um auxiliar de enfermagem e em média cinco agentes comunitários de saúde
(ACS). Uma das equipes atendia a comunidade de Airões, realizando ações individuais (consultas, curativos, exames, pré-natal, puericultura, vacinas), visitas domiciliares e al gumas atividades em grupo (palestras e mutirões) sobre educação em saúde, enfocando causas, consequência e principais formas de prevenção e tratamento de doenças ${ }^{12}$.

Evolução dos indicadores de saúde da população

Considerando as informações sobre as morbidades, enquanto em 1992 foi relatada a ocorrência de pelo menos uma enfermidade em $49,6 \%$ das famílias, em 2003, estenúmero aumentou para63\% e o total de pessoas enfermas passou de 89 em 1992 para 125 em 2003. A distribuição dos enfermos por sexo foi semelhante nos dois períodos, havendo variação entre as faixas etárias (Tabela 1 ).

O Gráfico 1 apresenta a distribuição percentual das enfermidades por grupos de causas em 1992 e2003.

Considerando o tipo de assistência escolhida para o tratamento das enfermidades, a procura por serviços do SUS diminuiu de 84,1\% em 1992 para $79 \%$ em 2003 , dos quais $26,7 \%$ foram realizados pelo PSF em 2003. A procura por atendimento particular epor convênio de saúde privados aumentou de $5,7 \%$ para $12,3 \%$ e de $2,3 \%$ para $3,4 \%$, respectivamente, enquanto que o tratamento realizado em casa e a procura pelo farmacêutico diminuíram.

0 número de internações reduziu de $42,7 \%$ no ano de 1992 para 27,2\% em 2003 e o tempo de internação também foi maior em 1992, uma vez
Tabela 1. Distribuição dos enfermos por sexo e faixa etária. Airões, Paula Cândido, M inas Gerais, Brasil, 1992 e 2003.

\begin{tabular}{lrrrr}
\hline \multirow{2}{*}{ Característica } & \multicolumn{2}{c}{1992} & \multicolumn{2}{c}{2003} \\
\cline { 2 - 5 } & $\mathrm{n}$ & \multicolumn{1}{c}{$\%$} & $\mathrm{n}$ & $\%$ \\
\hline Sexo & & & & \\
$\quad$ M asculino & 40 & 45 & 53 & 42,4 \\
$\quad$ Feminino & 49 & 55 & 72 & 57,6 \\
Faixa etária (anos) & & & & \\
$\quad<1$ & 02 & 2,2 & 01 & 0,5 \\
$1-10$ & 21 & 22,3 & 21 & 11,5 \\
$10-20$ & 09 & 9,6 & 07 & 3,8 \\
$20-60$ & 36 & 38,3 & 76 & 41,6 \\
$\geq 60$ & 26 & 27,6 & 18 & 42,6
\end{tabular}

Fonte: Inquéritos domiciliares realizados em 1992 e 2003.

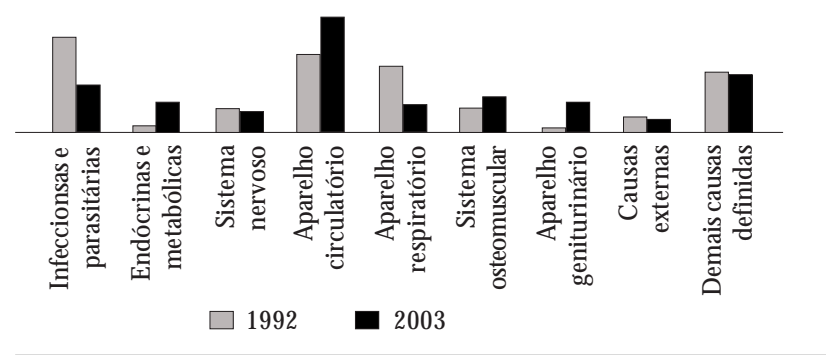

Gráfico 1. Distribuição percentual das enfermidades por grupos de causas. Airões, Paula Cândido, M inas Gerais, Brasil, 1992 e 2003.

Fonte: Inquéritos domiciliares realizados em 1992 e 2003. 
que neste ano 57,9\% dos doentes ficaram internados mais que setedias, enquanto que em 2003 apenas $23,5 \%$ dos doentes permaneceram no hospital mais que uma semana.

A distribuição percentual das internações por grupos de causas entre os períodos avaliados demonstrou redução nas internações por doenças infecciosas e parasitárias ( $16,7 \%$ para $6,7 \%)$, doenças do sistema nervoso ( $11,1 \%$ para $4,4 \%$ ), doenças do aparelho respiratório ( $27,8 \%$ para $15,5 \%$ ) e elevação por doenças do aparelho circulatório (13,9\% para $26,7 \%)$, doenças do aparelho osteomuscular (2,8\% para $8,9 \%$ ) e causas externas $(5,5 \%$ para 6,7\%).

Em relação aos óbitos, em 1992 foram relatados 15 óbitos, enquanto em 2003 este número aumentou para 67, com distribuição semel hante entre os sexos e variação em relação à faixa etária (Tabela 2).

As principais causas deóbitos referentes ao ano de 2003 estão especificadas no Gráfico 2 ea Tabela 3 apresenta o coeficiente de mortalidade para algumas causas selecionadas nos dois momentos do estudo.

Considerando a assistência pré-natal, enquanto apenas 30,6\% das gestantes em 1992 realizaram 0 pré-natal, em 2003, a coberturaaumentou para $90 \%$ das gestantes. Em 1992, 51,2\% das mães afirmaram ter apresentado algum problema durante a gravidez, prevalência que reduziu para $25,3 \%$ em 2003, e os principais problemas relatados nos dois períodosforam enjôo/mal-estar, edema, hipertensão, pré eclâmpsia, anemia, obesidade e hemorragia.

Em relação à incidência de abortos, $37,2 \%$ das mães em 1992 sofreram pelo menos um aborto e, em 2003 , a incidência caiu para $30,2 \%$, sendo as

Tabela 2. Distribuição dos óbitos por sexo e faixa etária. Airões, Paula Cândido, M inas Gerais, Brasil, 1992 e 2003.

\begin{tabular}{lrrrr}
\hline \multirow{2}{*}{ Característica } & \multicolumn{2}{c}{1992} & \multicolumn{2}{c}{2003} \\
\cline { 2 - 5 } & \multicolumn{1}{c}{$\mathrm{n}$} & \multicolumn{1}{c}{$\mathrm{n}$} & $\%$ \\
\hline Sexo & & & & \\
$\quad$ Masculino & 10 & 66,7 & 43 & 64,2 \\
$\quad$ Feminino & 05 & 33,3 & 24 & 35,8 \\
Faixa etária (anos) & & & & \\
$\quad<1$ & 01 & 6,7 & 12 & 17,9 \\
$1-10$ & 03 & 20 & 09 & 13,4 \\
$10-20$ & - & - & 01 & 1,5 \\
$20-60$ & 08 & 53,3 & 19 & 28,4 \\
$\geq 60$ & 03 & 20 & 26 & 38,8
\end{tabular}

Fonte: Inquéritos domiciliares realizados em 1992 e 2003. causas relatadas acidente ou queda, problemas de saúde da mãe, esforço físico, contrariedade ou susto e má-formação.

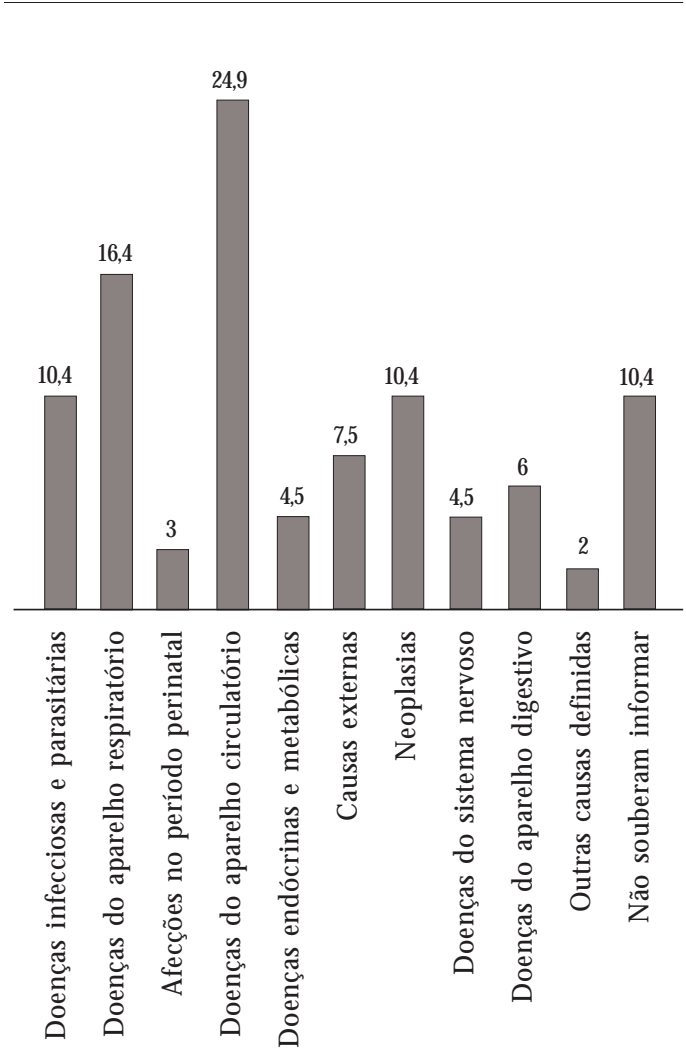

Gráfico 2. M ortalidade proporcional segundo grupo de causas. Airões, Paula Cândido, M inas Gerais, Brasil, 2003.

Fonte: Inquéritos domiciliares realizados em 2003.

Tabela 3. Coeficiente de M ortalidade para algumas causas selecionadas. Airões, Paula Cândido, Minas Gerais, Brasil, 1992 e 2003.

\begin{tabular}{lrc}
\hline \multicolumn{1}{c}{ Característica } & 1992 & 2003 \\
\hline N eoplasias & - & 38,7 \\
Doenças infecciosas e parasitárias & 20 & 38,7 \\
Doenças do aparelho circulatório & 33,3 & 88,6 \\
Afecções período perinatal & - & 14,6 \\
Doenças do aparelho respiratório & 6,6 & 60,9 \\
Causas externas & 6,6 & 27,7 \\
Diabetes & - & 16,6 \\
& &
\end{tabular}

Fonte: Inquéritos domiciliares realizados em 1992 e 2003. 
A incidência de filhos nascidos mortos também reduziu de 18,8\% em 1992 para 10,7\% em 2003. Quando questionadas sobre as causas da morte, a maioria das mães não soube informar; entre as causas relatadas, destacaram-se problemas de saúde da mãe, queda, esforço físico, problemas congênitos, agressão física, contrariedade e atraso no parto.

Analisando as características denascimento dos filhos, quanto ao atendimento elocal derealização do parto, houveuma grandeevolução de 1992 para 2003. Enquanto 48,9\% dos partos em 1992 foram realizados no domicílio, $99 \%$ dos partos acontece ram no hospital em 2003; entretanto, a cesariana aumentou de 16,3\% em 1992 para 45,7\% em 2003.

Com relação à prematuridade e ao baixo peso ao nascer, ambos apresentaram uma pequena elevação em suas incidências de 1992 para 2003, aumentando de $6,7 \%$ para $7,1 \%$ e $10 \%$ para $12,2 \%$, respectivamente.

\section{Discussão}

Considerando a taxa de cobertura do PSF, Paula Cândido apresentou maior cobertura em todos os períodos avaliados, uma vez que os municípios com população inferior a 20 mil habitantes apresentaram percentuais de cobertura de $9,41 \%$ em 1998; 55,99\% em 2002 e $61,6 \%$ em $2003^{16}$.

Analisando as enfermidades eas principaiscausas de internação hospitalar na população de Airões, tanto a redução verificada por doenças do sistema nervoso e do sistema respiratório quanto a elevação por doenças do aparelho circulatório e causas externas também foram verificadas no Brasil. Por outro lado, enquanto as doenças infecciosas e parasitárias diminuíram consideravelmente na comunidade, o país apresentou uma pequena elevação em suas taxas ${ }^{17}$.

Estas mudanças podem estar relacionadas às intensas transformações experimentadas pelo Brasil, a partir da segunda metade do século XX, em sua estrutura populacional e no padrão de morbimortalidade, resultantes da constante queda da taxa de natalidade e, ainda, devido ao aumento da expectativa de vida ao nascer ${ }^{5,16}$.

Em consequência, doenças queacometiam mais a população infantil, como as infecciosas e parasitárias, apesar depersistirem, vão diminuindo em patamar de prioridade para as doenças crônico-degenerativas mais incidentes na população adulta eidosa, que atualmente vêm assumindo um importante papel no quadro de morbimortalidade brasileiro ${ }^{16,18}$.

Além disso, alguns agravos, como as doenças do aparelho respiratório, podem evoluir com qua- dros severos, mas são potencialmente reversíveis mediante ações por parte dos serviços de assistência à saúde ${ }^{5}$, como foi verificado nesteestudo, uma vez que o diagnóstico precoce, a busca ativa pelos profissionais e 0 acesso ao tratamento oferecido pelo PSF contribuíram para a redução desta enfermidade na comunidade de Airões.

Em relação à mortalidade, foi verificado um aumento notável no número deóbitos de 1992 para 2003, principalmente entre menores de 1 ano e maiores de 60 anos de idade, ao contrário do Brasil, que apresentou reduções de $11,1 \%$ da mortalidade geral e de $65 \%$ dos óbitos entre os menores de 10 anos verificadas entre 1980 e $200^{19}$.

Este resultado pode estar relacionado às inadequadas condições sanitárias en contradas na comunidade, uma vez que as principais causas dos óbitos nesta idade foram as doenças infecciosas e parasitárias (41,7\%) e que em 2003 somente 52,3\% dos domicílios tinham abastecimento deágua pela rede pública, $68,5 \%$ possuíam cobertura de rede de esgoto eapenas $13,9 \%$ tinham coleta pública delixo ${ }^{12}$.

A discussão dos dados desagregados por situação rural e urbana reafirma a distribuição desigual da infraestrutura em saneamento no país, apresentando pouco aumento na cobertura destes serviços nas áreas rurais, o que sem dúvida reflete na mortalidadeinfantil eem outras causas de morte e adoecimento 5,19 .

Além disso, outrajustificativa para esteaumento seria as fal has no registro dos óbitos na comunidade de Airões em 1992, pois um dos grandes problemas que ainda permeiam as análises de mortalidade no Brasil é o subregistro de óbitos, devido à ocorrência de sepultamentos sem a exigência de certidão, com magnitude expressiva em regiões pobres, dezona rural e entreos menores de 1 ano de idade ${ }^{19}$.

Entre 1980 e 2005, em geral, foram observadas quedas importantes nas taxas de mortalidade pelos vários grupos de causa, destacando-se as reduções nos grupos das doenças infecciosas, doenças cardiovasculares e doenças do aparelho respiratório, enquanto o grupo das neoplasiasfoi o queapresentou crescimento mais acentuado, evidenciando 0 aumento da carga global de câncer no Brasi $\left.\right|^{5,20}$.

Apesar do aumento verificado entre 1992 e 2003 para todas as causas de mortalidade na comunidade deAirões, os coeficientes foram menores que os apresentados pelo Brasil, cuja avaliação demonstrou uma elevação de 152,4 para 158,4 e 69,5 para 72,7 nos coeficientes de mortalidade por doenças do aparelho circulatório e causas externas, respectivamente e no mesmo período ${ }^{20,21}$.

É interessante observar a distribuição das doenças e mortes entre os sexos, uma vez que em 
ambos os momentos do estudo as doenças foram mais frequentes entre as mulheres e as mortes entre os homens. Desde o censo demográfico de 1991, esta diferença vinha sendo apresentada pela maior expectativa de vida das mulheres em relação aos homen $\mathrm{s}^{18}$, tendência que foi confirmada pelos dados do censo de 2000, quando o envelhecimento populacional mostrou um excedente feminino ${ }^{22}$.

E ainda verifica-se que as causas externas de mortalidade, como acidentes de trânsito, homicídios e acidentes de trabal ho, que afetam particularmente indivíduos jovens e do sexo masculino, são igualmente crescentes no país, acentuando os diferenciais por sexo $0^{18,19}$.

Q uando analisamos as internações, a incidência e o tempo de permanência no hospital sofreram reduções consideráveis entre 1992 e 2003, o que pode ser atribuído ao maior acesso aos serviços de saúde com o PSF, uma vez que a taxa de internação por doenças crônico-degenerativas na população acima de 40 anos reflete indiretamente a disponibilidade de ações básicas de prevenção e controle da doença hipertensiva ${ }^{16,23}$.

Roncalli et al. ${ }^{9}$ concluíram que a implantação do PSF não contribuiu para a queda nas taxas de hospitalização em municípios da Região N ordeste, ao contrário da redu ção verificada pelo país nas taxas de internação por acidente vascular cerebral e insuficiência cardíaca congestiva, ocorrendo declínio mais intenso nos estratos com maior cobertura do PSF ${ }^{16,17}$.

Considerando o tipo de assistência recebida pelas pessoas enfermas, a procura pelo atendimento particular e por convênios de saúde privados apresentou um aumento respectivo de $340 \%$ e $200 \%$ de 1992 para 2003.

De acordo com os autores Cotta et al. ${ }^{24}$, como nas outras esferas da sociedade brasileira, o sistema de saúde se privatiza pela forma desorganizada (intencional ou não) de implantação das reformas pelo governo, deixando todos os espaços possíveis para que os planos de saúde privados pareçam evoluir naturalmente e para que o Estado se capacite a atender mais eficientemente os setores sociais de menor poder aquisitivo.

Por outro lado, a referência ao PSF por apenas $26,7 \%$ dos entrevistados demonstra o desconhecimento e a inadequada percepção dos usuários quanto ao programa, uma vez que as ações de saúde desenvolvidas na comunidade de Airões estavam totalmente vinculadas ao PSF e que o programa cobria quase $100 \%$ da comunidade em 2003.

Realidade semel hantefoi verificada no município de Teixeiras (MG), no qual $78,6 \%$ dos usuários não souberam ou não responderam as perguntas sobre o PSF. Tais resultados são preocupantes, pois se a comunidadeéa razão da existência do sistema de saúde, o conhecimento e a percepção dos usuários sobre seus princípios e diretrizes é de suma importância para a construção da consciência sanitária e o cumprimento do seu papel de cidadão na luta por seus direitos e pela melhoria da atenção a saúde ${ }^{25}$.

Em relação à assistência prénatal, considerando que 0 acompanhamento da gestante permite 0 diagnóstico e tratamento de inúmeras complicações durante a gravidez, favorecendo a redução ou eliminação de fatores de risco passíveis de serem corrigidos ${ }^{19}$, os resultados sugerem a melhoria da atenção pré-natal em Airões com a atuação do PSF, principalmente pelo aumento da cobertura das gestantes, que foi semelhante à nacional em $2003^{16,19}$

Os estudos em geral avaliam como de impacto positivo a implantação do PSF sobre o número de consultas e a qualidade do pré-natal ${ }^{7,9,16,23,26}$, contribuindo para a melhoria do acesso das gestantes ao atendimento pré-natal, aumentando a cobertura vacinal e o número de consultas 8,26 .

Em M ontes Claros, pesquisa comparando a Estratégia Saúde da Família com centros de saúde tradicionais demonstrou que o processo da assistência à saúde materno-infantil é mais adequado no modelo do PSF, apresentando melhor desempenho nas ações de orientação sobre aleitamento materno, orientações nutricionais para a gestante e realização de exame de prevenção do câncer de colo uterino durante o pré natal ${ }^{27}$.

Quanto às características de nascimento dos filhos, verificamos uma evolução na assistência ao parto, já que a maioria passou a ser realizada no hospital, corroborando Andrade et al ${ }^{23}$. Entretanto, o número de cesáreas aumentou de 16,3\% para $45,7 \%$, proporção semel hante à encontrada na Região Sudeste $(46,9 \%)$ eque contraria recomendação da Organização Mundial da Saúde de que partos cesáreos não ultrapassem $15 \%$ do total de nascimentos ${ }^{28,29}$.

Estudo realizado pela Agência N acional deSaúde Suplementar verificou que, em 2006, as taxas de cesarianas no Brasil chegaram a 80,5\% nos hospitais particulares, uma proporção que se configura como grave problema de saúde pública, pois aumenta os riscos da ocorrência de eventos relacionados à morbimortalidade materna e neonata ${ }^{29}$.

Com relação ao baixo peso ao nascer eà prematuridade, foi observado um pequeno aumento em suas incidências no período do estudo, mas as pre valências ficaram próximas às do Brasil. Contudo, a prematuridade associada ao baixo peso ao nascer 
são condições que conferem maiores riscos demortalidadeedecomprometimento da qualidadedevida de muitos dos que sobrevivem, sendo considerados importantes questões de saúde pública e que merecem maior atenção dos serviços de saúde ${ }^{30}$.

Considerando que o número de consultas pré natais éfator de risco para o baixo peso e a prematuridade e que 0 aumento da cobertura pré natal pode proporcionar resultados imediatos para a redução do baixo peso e/ou prétermo ${ }^{31}$, a elevação verificada nas prevalências destes agravos em Airões possivelmente está relacionada ao subre gistro das informações em 1992, devido à alta pre valência de partos domiciliares.

A Pesquisa Nacional de Demografia e Saúde ${ }^{28}$ verificou a ocorrência de partos domiciliares nas regiões rurais em condições precárias e com ausência de notificação aos sistemas de informação em saúde. Além disso, a comparação do número de nascimentos ocorridos nos hospitais do SUS e os declarados no Sistema de Nascidos Vivos (SINASC) ainda mostra deficiência no registro e na cobertura do SINASC em al guns estados do país ${ }^{19}$.

Por outro lado, esta elevação pode indicar assistência pré-natal de baixa qualidade, pois apesar da ampliação da cobertura verificada no Brasil, $52 \%$ do total de mortes em crianças menores de 1 ano ocorreram no período neonatal eas principais causas estavam associadas à baixa qualidade da assistência à gestação, ao parto e ao puerpério ${ }^{19,32}$.

Em São Paulo, foram registradas taxas de 8,8\% para o baixo peso, 7,3\% para a prematuridade e de $12 \%$ para a prevalência de baixo peso e/ou prematuridade entre os nascidos vivos ${ }^{31}$, enquanto que em Goiânia a prematuridade foi responsável por $47,2 \%$ dos casos de baixo peso ao nascer, confirmando a intensa relação entre estes dois fatores ${ }^{33}$.

Já em I taúna (M G), embora as taxas de baixo peso $(7 \%)$ e prematuridade $(5,3 \%)$ tenham sido mais baixas, a análise dos fatores determinantes foi prejudicada devido aos erros e/ou ausência de dados nas declarações de nascimentos, demonstrando que apesar dos avanços já alcançados, a manutenção de dados ignorados para al gumas informações ainda é uma situação real e que precisa ser modificada ${ }^{34}$.

De maneira geral, os resultados encontrados neste estudo demonstram a ampliação da cobertura de serviços na atenção básica a partir da expansão do PSF, assim como a contribuição positiva do programa na evolução de muitos dos indicadores de saúde da população. Todavia, algumas ações e serviços carecem de melhorias para garantir uma assistência integral e de maior qualidade aos usuários.
Vários são os estudos quedemonstram a eficiência do PSF a partir dos impactos positivos produzidos nos indicadores de saúde da população e da garantia de maior equidade em saúde no país mas, ao mesmo tempo, constatam que a estratégia ainda está em construção e necessita de qualificação para concretizar a mudança do modelo assistencial e obter resultados mais expressivos $23,3,-9,23,27,35$.

Além disso, o aumento da cobertura dos serviços do PSF associados aos esforços deliberados para melhorar os sistemas de informações em saúde já produziu as vantagens possíveis na saúde dos brasileiros, de forma que, sem reduções significativas nas iniquidades sociais, será impossível avançar de maneira mais substancial no quadro global de saúde da população ${ }^{5}$.

N este sentido, a articulação com outras políticas públicas adquire relevância ímpar, sem a qual serão mantidas as desigualdades nos padrões epidemiológicos atuais, uma vez que determinadas situações de saúde se apresentam como resultado das condições concretas de existência sobreas quais os serviços de saúde, quaisquer que sejam, possuem limitada capacidade de impacto ${ }^{9}$.

Tais políticas, necessariamente, devem ser dirigidas para os determinantes do processo saúdedoença, visando ao enfrentamento da complexidade de sua produção a partir de ações intersetoriais. Além disso, devem estimular a conscientização da população, para que gradativamente ela se torne ativa e parceira neste processo de mudança do sistema de saúde, cumprindo assim o seu papel e a democracia conquistada com a Constituição de 1988.

\section{Considerações finais}

Os resultados reforçam o potencial que o PSF possui para reestruturar o modelo de assistência à saúde, tornando realidade muitas das diretrizes do SUS. De fato, na comunidade de Airões, 0 acesso aos serviços e ações em saúde foi facilitado com a implantação do programa, refletindo na melhoria de muitos dos indicadores de saúde da população.

Entretanto, a maioria das ações realizadas pelo programa ainda seguiu o modelo tradicional de assistência, priorizando ações curativas e individualizadas, o quelimitou seu potencial demudança. Este desafio foi retratado pelos indicadoresquenão apresentaram melhoria após sua implantação e principalmente pelo fato da maior parte dos usuários desconhecerem a existência do próprio programa.

N este sentido, para que o PSF seja capaz de priorizar as ações de promoção da saúde e prevenção de agravos, seus princípios e diretrizes devem 
ser apresentados insistentemente aos profissionais de saúde e aos usuários, para que juntos e orientados pela nova lógica do sistema de saúde, el es possam desempenhar os seus papéis na efetiva implementação de um serviço de saúde universal, equitativo, democrático, resolutivo e de qualidade.

\section{Colaboradores}

KO Gomes participou da coleta e análise dos dados e redação do artigo. RM M Cotta participou da idealização, delineamento de objeto de estudo, desenho da metodologia e foi responsável pela coordenação, orientação e acompanhamento do trabalho de campo, da redação e revisão do artigo. CL Targueta participou da idealização, delineamento do objeto de estudo, desenho da metodologia, coleta e processamento dos dados. SE Prioree SCC Franceschini participaram da ideal ização, delineamento do objeto de estudo, desenho da metodologia, análise dos dados e revisão crítica do artigo. M P Euclydes participou da idealização, delineamento do objeto de estudo e revisão critica do artigo.

\section{Agradecimentos}

Os autores agradecem aos ACS de Airões eà Prefeitura Municipal de Paula Cândido pelo apoio oferecido para a realização do trabalho, assim como às famílias entrevistadas pela receptividade.

\section{Referências}

1. Brasil. Ministério da Saúde. Programa Saúde da Família. Brasília: M inistério da Saúde; 2001.

2. Brasil. Ministério da Saúde. Avaliação normativa do Programa Saúde da Família no Brasil: monitoramento da implantação e funcionamento das equipes de saúde da família: 2001-2002. Brasília: M inistério da Saúde; 2004.

3. Brasil. Ministério da Saúde. Fundação Oswaldo Cruz. Saúde da Família: avaliação da implementação em dez grandes centros urbanos: síntese dos principais resultados. 2a ed. atual. Brasília: Ministério da Saúde; 2005.

4. Sousa M F, Hamann EM. Programa Saúde da Família no Brasil: uma agenda incompleta? Cien Saude Colet 2009: (14) 5:1325-1335.

5. Barreto ML, Carmo EH. Padrões de adoecimento e de morte da população brasileira: os renovados desafios para o Sistema Ú nico de Saúde. Cien Saude Colet 2006; 12(Supl.):1779-1790.

6. Silva AC. 0 impacto do Programa de Saúde da Família no município de Sobral - Ceará: uma análise da evolução da saúde das crianças menores de cinco anos de idade no período de 1995-2002 [dissertação]. São Paulo (SP): Faculdade de M edicina, U niversidade de São Paulo; 2003.

7. Szwarcwald CL, M endonça M HM, Andrade CLT. Indicadores de atenção básica em quatro municípios do Estado do Rio de Janeiro, 2005: resultados de inquérito domiciliar de base populacional. Cien Saude Colet 2006; 11(3):643-655.

8. Facchini LA, Piccini RX, Tomasi E, Thumé E, Silveira DS, Siqueira FV, Rodrigues MA. Desempenho do PSF no Sul e no Nordeste do Brasil: avaliação institucional e epidemiológica da atenção básica à saúde. Cien Saude Colet 2006; 11(3):669-681.

9. Roncalli AG, Lima KC. Impacto do Programa Saúde da Família sobre indicadores de saúde da criança em municípios de grande porte da região Nordeste do Brasil. Cien Saude Colet 2006; 11(3):713-724.

10. Azevedo G, M endonça S. Principais desafios para os inquéritos populacionais na organização de um Sistema Integrado de Informação em Saúde. Cien Saude Colet 2006; 11(4):870-886.

11. Santos GF. Representação social do processo saúde/doença na comunidade rural de Airões: Zona da M ata de M inas Gerais [dissertação]. Viçosa (M G): Universidade Federal de Viçosa; 1992. 
12. Gomes KO, Cotta RM M, Priore SE, Euclydes M P, Franceschini SCC. Saúde e nutrição da população rural de Airões, município de Paula Cândido, MG: avaliação do impacto do Programa Saúde da Família (PSF) no perfil epidemiológico da população e diagnóstico de anemia ferropriva em crianças de 0 a 60 meses. In: Brasil. Ministério da Saúde. Prêmio de Incentivo em Ciência e Tecnologia para o SUS: 2007. Brasília: Ministério da Saúde; 2007.

13. Instituto Brasileiro de Geografia e Estatística. Síntese de Indicadores Demográficos. [site da Internet] 2006 [acessado 2006 mar 23]; [cerca de 4 p.] Disponível em: http://www.ibge.gov.br/cidadesat/xtras/perfil.php

14. Richardson RJ. Pesquisa social: métodos e técnicas. 3a ed. São Paulo: Atlas; 1999.

15. Richardson RJ. Pesquisa social: métodos e técnicas. $2^{\underline{a}}$ ed. São Paulo: Atlas; 1989.

16. Brasil. M inistério da Saúde. Saúde da Família no Brasil: uma análise de indicadores selecionados: 1998-2004. Brasília: M inistério da Saúde; 2006.

17. Departamento de Informática do SUS. Indicadores e Dados Básicos - Brasil - 2006 IDB-2006. Proporção de internações hospitalares (SUS) por grupos de causas. [site da Internet] 2008 [acessado 2008 abr 14]; [cerca de 15 p.] Disponível em: http://tabnet.datasus.gov.br/ cgi/idb2006/Grupo_D.xls

18. Patarra NL. Mudanças na dinâmica demográfica. In: M onteiro CA, organizador. Velhos e novos males de saúde no Brasil: a evolução do país e de suas doenças. São Paulo: Hucitec; 1995. p. 61-89.

19. Brasil. M inistério da Saúde. Saúde Brasil 2004 - uma análise da situação de saúde. Brasília: Ministério da Saúde; 2004.

20. Departamento de Informática do SUS. Indicadores e Dados Básicos - Brasil - 2006 IDB-2006. Indicadores de M ortalidade. [site da Internet] 2008 [acessado 2008 abr 13]; [cerca de 15 p.] Disponível em: http:// tabnet.datasus.gov.br/cgi/idb2006/Grupo_C.xls.

21. Rede Interagencial de Informações para a Saúde. Indicadores básicos de saúde no Brasil: conceitos e aplicações. Brasília: Organização Pan-Americana da Saúde; 2002.

22. Instituto Brasileiro de Geografia e Estatística. 2006. Sistema Nacional de Informações de Gênero (SNIG). [site da internet] 2006 [acessado 2006 mai 25]; [cerca de 8 p.]. Disponível em: http://www.ibge.gov.br/muIheres/alvo//políticas/públicas.php

23. Andrade LOM, Barreto ICHC, Goya N, Júnior TM Organização da atenção básica e Estratégia Saúde da Família no município de Sobral - Ceará - Brasil: principais avanços e desafios na perspectiva de produzir mudanças positivas na saúde. In: Brasil. Ministério da Saúde. Experiências e Desafios da Atenção Básica e Saúde Familiar: caso Brasil. Brasília: M inistério da Saúde; 2004. p.143-179.

24. Cotta RM M, Mendes FF, Muniz JN. A crise do SUS e a fuga para o mercado: o seguro-saúde e a mercantilização da Saúde. In: Cotta RM M, organizador. Descentralização das políticas públicas de saúde: do imaginário ao real. Viçosa: Editora UFV; 1998. p. 125-142.
25. Cotta RM M, Azeredo CM, Schott M, Cardoso PM, Franceschini SCC, Priore SE. Sobre o conhecimento e a consciência sanitária brasileira: o papel estratégico dos profissionais e usuários no sistema sanitário. Revista M édica M inas Gerais 2006; 16(1):2-8.

26. Moura ERF, Holanda Jr. F, Rodrigues M SP. Avaliação da assistência pré-natal oferecida em uma microrregião de saúde do Ceará, Brasil. Cad Saude Publica 2003; 19(6):1791-1799.

27. Caldeira AP, Oliveira RM, Rodrigues. Qualidade da assistência materno-infantil em diferentes modelos de atenção primária. Cien Saude Colet [periódico na Internet] 2008. [acessado 2007 fev 21]; [cerca de 15 p.] Disponível em: http://www.abrasco.org.br/cienciaesaudecoletiva/artigos/artigo_int.php?id_artigo $=1525$

28. Brasil. M inistério da Saúde. Política Nacional de Atenção Integral à saúde da Mulher: Princípios e Diretrizes. Brasília: Ministério da Saúde; 2004.

29. Estudo detalha riscos da cesárea. Radis - Comunicação em Saúde 2008; 66:7.

30. Audi CAF, Corrêa MAS, Latorre M RDO, Santiago SM . Associação entre violência doméstica na gestação e peso ao nascer ou prematuridade. J. Pediatr. (Rio J.) $2008 ; 84(1): 60-67$

31. Kilsztajn S, Rossbach A, Carmo M SN, Sugahara GTL. Assistência pré-natal, baixo peso e prematuridade no Estado de São Paulo, 2000. Rev. Saude Publica 2003; 37(3):303-310

32. Brasil. M inistério da Saúde. Saúde da Família e a Atenção PréN atal e Puerperal. Brasília: M inistério da Saúde; 2006.

33. Giglio M RP, Lamounier JA, N eto OLM, César CC. Baixo peso ao nascer em coorte de recém-nascidos em Goiânia-Brasil no a no de 2000. Rev Bras Ginecol Obstet. 2005; 27(3):130-136.

34. Guimarães EAA, Velásquez-M eléndez $G$. Determinantes do baixo peso ao nascer a partir do Sistema de Informação sobre Nascidos Vivos em Itaúna, M inas Gerais. Rev. Bras. Saude M ater. Infant. 2002; 2(3):283290.

35. Henrique F, Calvo M CM . Avaliação do Programa Saúde da Família nos municípios do Estado de Santa Catarina, Brasil. Cad Saude Publica 2008; 24(4):809-819.

Artigo apresentado em 13/06/2007

Versão final apresentada em 30/06/2007 